\title{
Polyelectrolyte Bundles
}

\author{
H. J. Limbach, M. Sayar, and C. Holm $\dagger$ \\ Max-Planck-Institut für Polymerforschung, Ackermannweg 10, 55128 Mainz, \\ Germany
}

\begin{abstract}
Using extensive Molecular Dynamics simulations we study the behavior of polyelectrolytes with hydrophobic side chains, which are known to form cylindrical micelles in aqueous solution. We investigate the stability of such bundles with respect to hydrophobicity, the strength of the electrostatic interaction, and the bundle size. We show that for the parameter range relevant for sulfonated poly-para-phenylenes (PPP) one finds a stable finite bundle size. In a more generic model we also show the influence of the length of the precursor oligomer on the stability of the bundles. We also point out that our model has close similarities to DNA solutions with added condensing agents, hinting to the possibility that the size of DNA aggregates is under certain circumstances thermodynamically limited.

E-mail: $\{$ limbach, sayar, holm\}@mpip-mainz.mpg.de
\end{abstract}

\section{Introduction}

Aggregates of charged semiflexible polymers have been experimentally observed in a variety of systems, where the most well known examples are probably toroidally shaped bundles in DNA solutions with added condensing agents, such as multivalent counterions [1, 2, 3. Another polyelectrolyte system of synthetic nature, consisting of poly(para-phenylene) (PPP) oligomers 4, 5, 6, which are short rodlike charged objects, forms cylindrical micelles due to its hydrophobic side chains. This model can be well controlled chemically, and can be used as a synthetic model system to study nano-aggregation of charged filaments. An additional advantage of PPPs is that they allow one to tune experimentally the electrostatic and hydrophobic interactions separately. Although the physical origin of both aggregation mechanisms is different, in a first approximation they can be regarded as being the result of a short range attraction. This interaction is, in the case of DNA, the result of short range ionic correlations of the multivalent counterions [7, whereas in the case of the PPPs it is due to short range interactions of the hydrophobic side chains. The aggregate size in both cases is determined by the competition between the surface tension (due to short range attractions), the repulsive self-energy of the backbone charges, and the entropic degrees of freedom of the counterions. For DNA it has been argued 8, 9, 10, 11] that the observed finite size of the DNA aggregates is due to kinetic problems, i.e. electrostatic barrier formation, and not set by thermodynamic properties of the system. Experiments performed with viral DNA support this conjecture at least for the investigated systems 12. However, recently Henle and Pincus 13 have argued, using a charged rod model interacting via a short range attraction, that, depending

$\dagger$ To whom correspondence should be addressed 
on the actual parameters of the system, either finite or infinite bundle sizes should be possible. Treating the systems as consisting of sticky charged rods brings up the analogy to the Rayleigh split of a charged hydrophobic droplet. This problem has been solved in a mean-field model already by Deserno [14. He observes that the droplet size is always finite, if the counterions cannot penetrate into the droplet, but that allowing counterion penetration leads either to finite or infinite droplet sizes, depending on the parameters. There have been relatively few simulations on bundle formations, notable exception being the simulations by Stevens [15] 16, showing the possibility that multivalent ions alone can lead to bundle formations, and the work of Borukhov et al. [17, which investigates two-rod systems bundled via short range mobile linker interactions. In the present work we investigate the bundle formation for a system of charged semi-flexible polymers with short range interactions for two models, and demonstrate clearly the existence of finite size aggregates in both cases.

\section{Simulation method}

\section{1. $P P P$}

The PPP oligomer is described with a bead spring model, whose mapping is shown in figure. 10 Each phenyl ring along the semi-flexible backbone is represented by a spherical bead. Two of them carry one negative unit charge $e_{o}$. The chain length

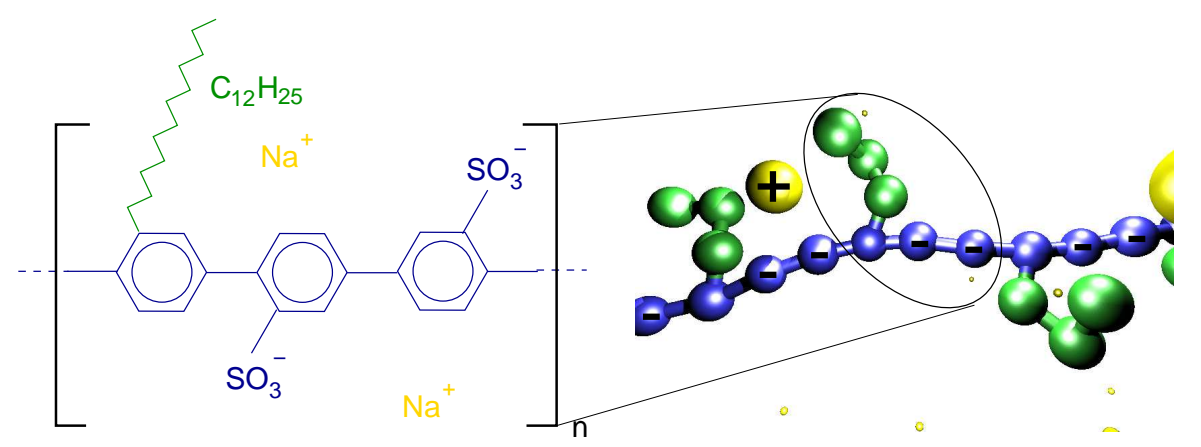

Figure 1. Mapping of a PPP monomer onto the bead spring model.

is $N_{m}=61$ beads representing $20 \mathrm{PPP}$ monomers. The hydrophobic side chain is modeled as a flexible chain containing 3 beads. The counterions are simulated explicitly as charged spheres carrying one unit charge. The solvent is taken into account implicitly represented as a background with dielectric constant $\epsilon$. The simulations are performed in an NVT ensemble. The temperature is maintained via a Langevin thermostat.

All particles interact via a repulsive Lennard-Jones potential with $r_{c u t}=2^{1 / 6} \sigma$ and $\epsilon_{L J}=1 k_{B} T$ (eq. 1) and charged particles interact via an unscreened Coulomb potential (eq. 22). Bonds are realized with a FENE [18 potential with $k_{F}=7 k_{B} T$ and a cutoff $R_{F}=2 \sigma$ (eq. 31). The PPP backbone is semi-flexible which is modeled with

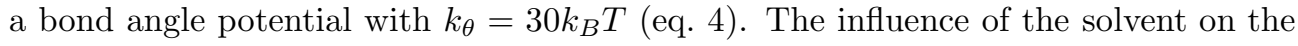
hydrophobic side chains is taken care of with an attractive Lennard-Jones potential 
between these monomers (eq. 11). Here the cutoff is $r_{c u t}=2.5 \sigma$.

$$
\begin{aligned}
& U_{L J}(r)=4 \epsilon_{L J}\left(\left(\frac{\sigma}{r}\right)^{12}-\left(\frac{\sigma}{r}\right)^{6}\right) \text { for } r<r_{c u t} \\
& U_{C}\left(r_{i j}\right)=\ell_{B} T \frac{q_{i} q_{j}}{r_{i j}} \\
& U_{F}(r)=\frac{1}{2} k_{F} R_{F}^{2} \ln \left(1-\left(\frac{r}{R_{F}}\right)^{2}\right) \text { for } r<R_{F} \\
& U_{\theta}(r)=k_{\theta}(1-\cos \theta)
\end{aligned}
$$

The parameters $\epsilon_{L J}$ for the attractive Lennard-Jones interaction and the Bjerrum length $\ell_{B}=e_{0}^{2} /\left(4 \pi \epsilon k_{B} T\right)$ have been varied. Due to the large kinetic barriers involved in the formation of a polyelectrolyte bundle, we study only its possible break up by starting the simulations with a preformed bundle in the middle of a spherical simulation cell. The cell radius is given by the particle density which is $\rho=1.0 \times 10^{-4} \sigma^{-3}$. The equilibration is done in a two step process. First we constrain the backbone monomers of the PPPs in space and let the hydrophobic hairs and counterions equilibrate for $100000 \mathrm{MD}$ steps. Then we release the backbone monomers and let the total system equilibrate. The bundle is simulated for $2000000 \mathrm{MD}$ steps. If the bundle is stable over the whole simulation time it is called stable in this paper. We are well aware that this procedure only proves that the observed bundles are at least in a metastable state and cannot prove that we are really in the global minimum of the free energy. However, we only want to demonstrate the existence of finite aggregate sizes, and therefore our method suffices, see e.g. Ref. [17] where the same simulation technique has been applied to the case of a two bundle system.

\subsection{Generic bundles}

Further simplification of this model can be achieved by taking the zero size limit of the hydrophobic side chains and making instead all backbone monomers hydrophobic, which is exactly the model Henle and Pincus suggested [13. Each bead carries a positive unit charge. The beads interact with all other charges via an unscreened Coulomb interaction (eq. 2). The beads are connected by a FENE potential (eq. 3) and furthermore the oligomer is semi-flexible, where the stiffness of the chain can be tuned by a bond angle potential (eq. 44). The hydrophobic interactions due to side chains are also represented via short range interactions of these beads, where the Lennard-Jones+Cosine potential (eq. 5) is used [19. This potential enables the use of large $\epsilon_{L J}$ values, while the function and it's first derivative are continuous at the cut off radius. The cut off radius is chosen to be extremely small, $r_{c u t}=1.5 \sigma$, so that the counterions can not penetrate into the bundle without breaking the short range interaction among the polyelectrolyte beads. All other interaction parameters are as described in section 2.1

$$
U_{C O S}(r)=\frac{\epsilon_{L J}}{2}\left(\cos \left(\alpha r^{2}+\beta\right)-1\right) \quad \text { for } \quad r_{\min } \leq r<r_{c u t}
$$




\section{Results and discussions}

\section{1. $P P P$}

For the PPP model we are interested in the influence of the hydrophobicity and the strength of the electrostatic interaction on the stability of the PPP micelles. We simulated PPP bundles of different aggregate sizes and varied systematically the parameters for the hydrophobicity $\epsilon_{L J}$ and the strength of the electrostatic interaction $\ell_{B}$. The simulated bundle sizes $N_{p}$ are 2, 3, 5, 8 and 10. In Figure 2 we show the stable and unstable regions in a phase diagram.



Figure 2. $\epsilon_{L J}-\ell_{B}$ phase diagram for bundles with $N_{p}=2,3,5,8,10$.

The phase boundary for bundles with different sizes are shown as lines connecting simulations where the bundles are marginally stable. Above the line bundles with that specific size are stable (actually, only at least meta-stable), below unstable.

Looking at the phase boundary for a given bundle size, e.g. $N_{p}=5$, with increasing $\ell_{B}$ the stability of the bundle first decreases. This means one needs a larger hydrophobicity to get a stable bundle. This can be explained by the increasing electrostatic repulsion of the charged backbones. At $\ell_{B} / \sigma \simeq 2$ the stability curve shows a maximum. Further increase of $\ell_{B}$ leads to more stable bundles. This nonmonotonic form resembles the behavior of the extension of flexible polyelectrolyte chains both in good 20, and poor solvent 21, 22, 23, 24. The increasing stability at high $\ell_{B}$ values is due to the increased density (condensation) of counterions close to the bundle which renormalize the effective charge and can also mediate attractive interactions at sufficiently large values of $\ell_{B}$.

In Figure 2 we can also compare the stability of bundles with different size. For $\ell_{B}=0.5 \sigma$ where the electrostatic interaction does not play a significant role, we see that for the investigated regime the range of $\epsilon$-values yielding stable bundles increases with increasing bundle size. This behavior changes drastically when one looks at $\ell_{B}$ values larger than $1.0 \sigma$. Here we find at $\epsilon$-values between 1.7 and 1.9 that bundles which contain between 5 and 8 PPPs are more stable than larger or smaller $N_{p}$ values. 
This implies also that one can find situations with a finite stable bundle size. Note that we observe this behavior in the relevant experimental region, namely $\ell_{B}=1.7 \sigma$ for PPP. We can explain this finding by an electrostatic instability in analogy to the Rayleigh instability of charged droplets 25, 14, which has been shown to be valid also for linear charged polymers 26, 27. When one further increases $\ell_{B}$, the marginal stable bundle size decreases more and more, and the stability lines for all bundles containing more than 3 PPPs merge. We expect that for even larger $\ell_{B}$ values one will find an infinite bundle size.

Finally we investigated the counterion distribution inside and in the close vicinity of a bundle. In Figure 3 we show a snapshot of a polyelectrolyte bundle together with its cross section. Note that the counterions are able to penetrate also inside the bundle. This is important for the theoretical approach to the Rayleigh instability of charged droplets as it is introduced by Deserno [14].

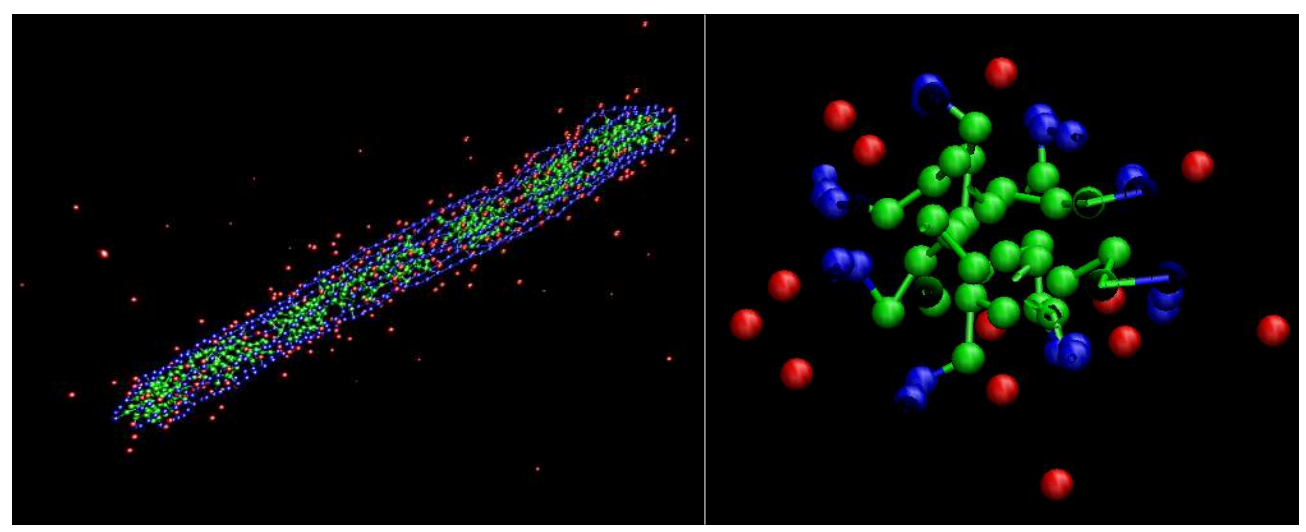

Figure 3. The snapshot of a polyelectrolyte bundle (left) together with a cross section (right) is shown for $N_{p}=8, \epsilon_{L J}=1.7 k_{B} T$ and $\ell_{B}=1.7 \sigma$

\subsection{Generic bundles}

Also for the generic bundle model as discussed above we find the formation of finite size aggregates. In order to study the contribution of various effects (e.g. strength of Coulomb and Lennard-Jones interactions, stiffness of the chains) the stability of a bundle of certain size can be tested as follows. A cylindrical bundle is preformed by aligning the backbone of the polymers along the axis of the cylinder. The crosssection of the bundle is chosen as a close-packed structure on a triangular lattice to minimize the surface area. Unlike the PPP polymers in the previous section, where the hydrophobic side chains where explicit, in this more generic model the hydrophobic interaction is also associated with the backbone beads. Therefore the polymers are not packed like a classical micelle. The lattice spacing for this initial configuration is chosen as the minimum of the Lennard-Jones + Cosine potential. The counterions are placed in a cylinder enclosing the bundle, where the radius of this cylinder is large enough to provide sufficient freedom during the relaxation of the counterions. The equilibration is started with the counterions, which are set free to move within this cylinder surrounding the bundle. However, it is important to note that the counterions cannot penetrate into the bundle at this stage due to the close 
packing of the polymers. After the counterions are equilibrated, the polymers are set free, and a brief equilibration run is performed where the polymers are also confined within a cylinder tightly enclosing the polymers, so that the bundle does not fall apart at this stage. Next the cylinder constraints are removed, and the system is integrated for $2.0 \times 10^{6}$ time steps. During the simulation, depending on the relative value of the interaction parameters, the bundle either remains as a single aggregate or splits up into smaller aggregates.

In Figure 5 the biggest stable bundle size obtained from the simulation is shown as a function of the number of polymers in the initial bundle. The polymers are $N_{m}=10$ beads long, and $\epsilon_{L J}=4.0 k_{B} T$ and $l_{B}=2.0 \sigma$. The stiffness of the chains is kept fixed $\left(k_{\theta}=10 k_{B} T\right)$. Up to six polymers per startup bundle, the structure does not disintegrate and the bundle remains as a single entity. However, for initial aggregates with more than $N_{p}=6$ polymers, the aggregate splits into two or more bundles. In Figure [5] the biggest size among these bundles is plotted. The stable equilibrium bundle size for this set of parameters is $N_{p} \approx 5$. However, due to the finite system size the equilibrium aggregate size obtained via this method must be taken cautiously.

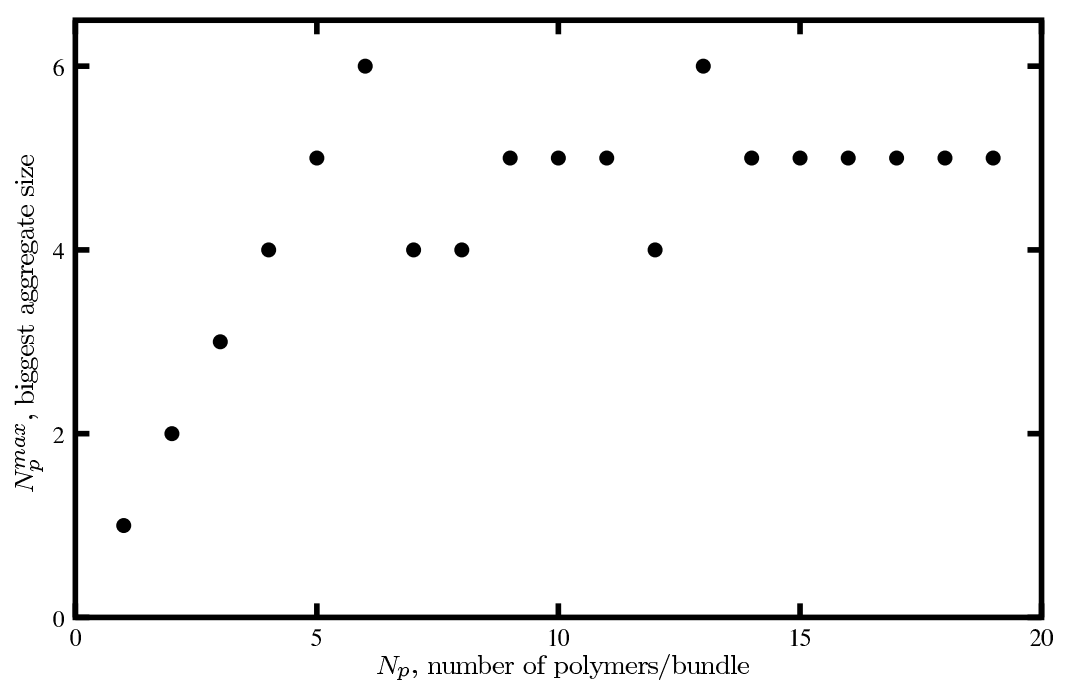

Figure 4. The biggest stable aggregate size $\left(N_{p}^{\max }\right)$ as a function of number of polymers $\left(N_{p}\right)$ in the startup bundle.

Another approach to obtain the average bundle size is to look at the internal energy of a bundle with counterions. The bundle is formed as in the previous case; however, in this simulation the bundle is constraint to remain as a single aggregate. In other words, the polymers are fixed throughout the simulation, and only the counterions are free to move. The average internal energy of the system as a function of the number of polymers per aggregate is plotted in Figure 5 for the same interaction parameters as in the previous case. In these constrained bundle simulations a minimum in the internal energy of the system is observed for $N_{p}=3$. Initially as the number of polymers per bundle increases, the internal energy of the system decreases as a result of decreasing surface energy. However, above the average aggregate size the electrostatic energy of the system increases dramatically. The energy distribution for bundles smaller than $N_{p}=7$ polymers is relatively flat. The sudden increase in the 
internal energy for the $N_{p}=7$ polymer aggregate is due to the complete isolation of the central polymer from the surrounding counterions. This is an artifact of fixing the polymers during the simulation. It is important to note that only the internal energy of the system is taken into account in this analysis. However, the entropy of the counterions could also play a dominant role in determining the average aggregate size for these polyelectrolytes with hydrophobic interactions. The slight difference in the equilibrium aggregate size obtained with these two methods presented above can be explained by the entropic contribution which is neglected in the fixed bundle simulations.

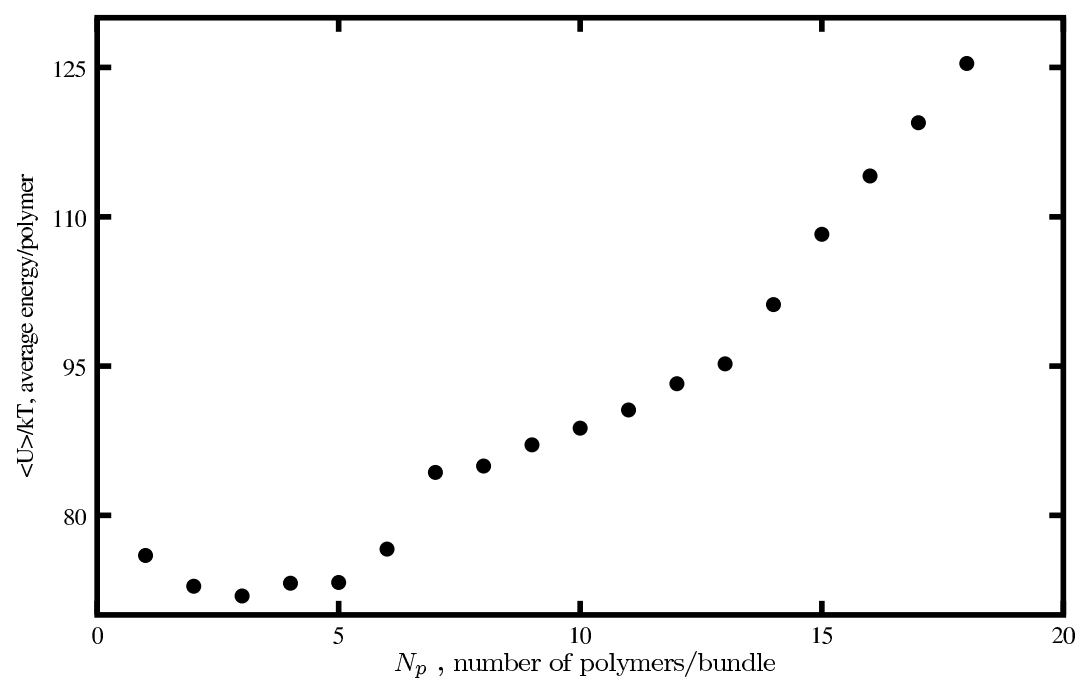

Figure 5. The average internal energy per polymer as a function of number of polymers $\left(N_{p}\right)$ in the startup bundle.

The length and charge density of the precursor polymers effect the fraction of condensed ions. Even though Poisson-Boltzmann theory yields a good approximation for the case of infinitely long polymers [28], for finite length rods there are no straightforward solutions. In order to gain further inside into the finite length effects, we have performed bundle simulations for lengths ranging from $N_{m}=10$ to $N_{m}=60$ monomers. Snapshots from the equilibrated systems of $N_{m}=20, N_{m}=40$, and $N_{m}=60$ are shown in Figure 6 For bundles with less than $N_{p}=6$ polymers all simulated polymer lengths yield stable aggregates, no splitting is observed. On the other hand, for bundles of 6 polymers even though the polymers of length $N_{m}=10$ and $N_{m}=20$ monomer length provide stable aggregates, for longer polymer lengths the bundle splits up.

The internal energy and the contributions from Coulombic and Lennard-Jones potentials per particle for bundles of $N_{p}=6$ polymers with polymer length of $N_{m}=60$ monomers is shown in Figure 7 Upon splitting, the internal energy of the bundle increases by almost one $k_{B} T$ per particle. The split takes place rather abruptly in a late stage of the simulation, which suggests the presence of a high energy barrier for the split. The split of the bundle into two small bundles is unfavorable in terms of the short range Lennard-Jones interactions since the surface energy increases. On the other hand for the Coulombic interactions the answer is more complicated. The electrostatic self energy of the bundle decreases due to split, since the like-charged 


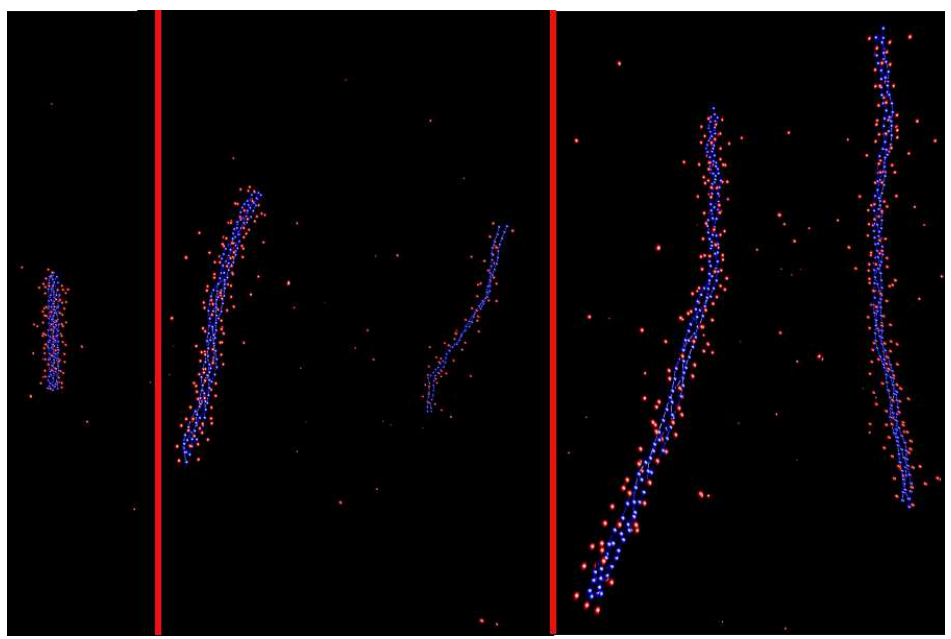

Figure 6. Snapshots from simulations of a 6 polymer bundle with polymer lengths $N_{m}=20, N_{m}=40$, and $N_{m}=60$ from left to right, respectively.

polymers repel each other. However, since the split-bundles attract less counterions the favorable electrostatic interactions among the polymers and counterions is also lost. For $N_{p}=6$ polymer bundle upon splitting the Coulombic contribution to the internal energy decreases.



Figure 7. The total internal energy (middle), Coulomb (top) and LennardJones (bottom) contributions per monomer for bundles of 6 polymers during the simulation.

The increase in the internal energy of the system can be advantageous for the system only if the release of the counterions sufficiently increases the entropy to compensate for the increase in internal energy. In Figure 8 the integrated counterion distribution for the $N_{p}=6$ polymer bundle is given for five time intervals during the 
simulation. For each counterion the distance to the closest monomer is chosen for the radial distribution function. Prior to the split (s0-s3) the counterion distribution does not change. Upon splitting up into two bundles the fraction of ions close to the bundles decreases dramatically. Therefore we can conclude that the splitting of these long polymer bundles are driven by the entropy of the released counterions.

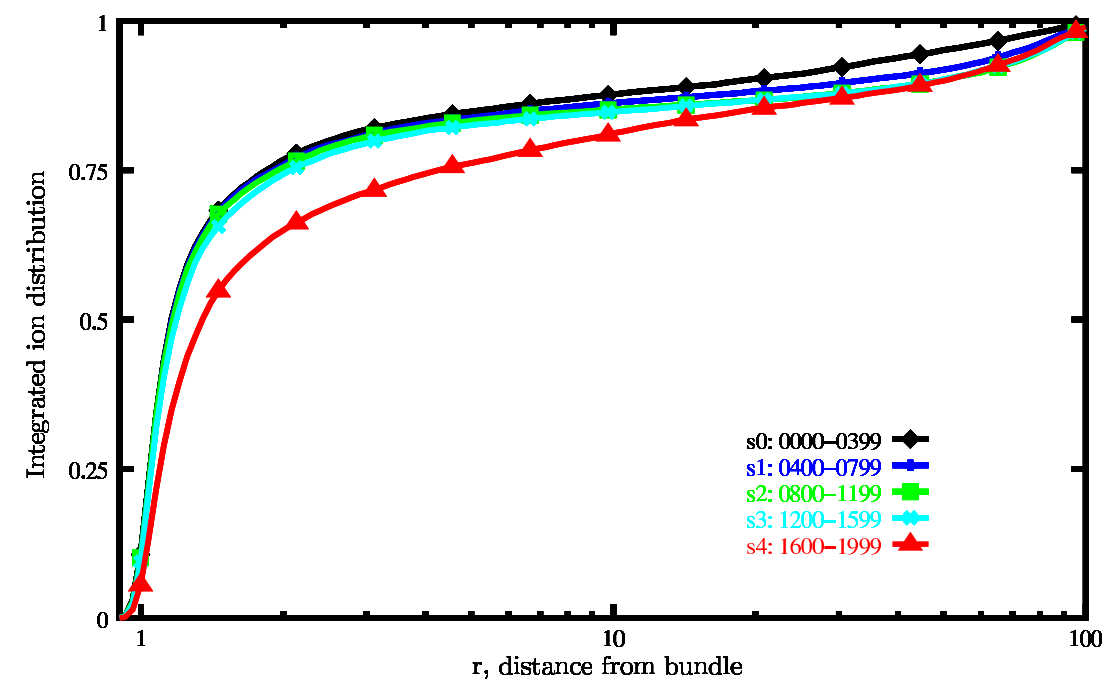

Figure 8. Integrated counterion distribution as a function of radial distance from the 6 polymer bundle. The distributions are calculated for five different time periods during the simulation. Time periods from top curve to the bottom one: s0 (0-399) - s4 (1600-1999). Time period s4 corresponds to the distribution after the bundle splits up.

\section{Conclusions}

We have shown that charged semi-flexible polyelectrolytes interacting via two kinds of hydrophobic short range interactions have parameter regions where finite aggregate sizes exist. For large values of $l_{B}$ the bundle size increases, and in the limit of large $l_{B}$ we observe the expected trend to form infinite bundles due to counterion crystallization. Furthermore, we could demonstrate that the release of counterions during a bundle split can considerably lower the free energy of the total system.

The stability curve for different bundle sizes as a function of $l_{B}$ shows similarities to the Rayleigh instability, i.e., for an increase in $l_{B}$ one needs an increased $\epsilon_{L J}$. Another observation is that the stability line varies non-monotonically with Bjerrum length, which resembles the non-monotonic extension behavior linear polyelectrolytes show if the Bjerrum length is increased. We relate this to the fact that the electrostatic self-energy of the bundle does not increase with $l_{B}$ after $l_{B} \approx 2 \sigma$ due to counterionbackbone ion correlations. The degree of polymerization shows also an effect on bundle size, which has been observed in experiments [4, 5] on PPPs as well; however, our limited data does not allow us to draw definite conclusions about the underlying mechanism.

One important point to note is the similarities of our model with the observed 
trends in DNA condensation experiments which also find finite aggregate sizes. The applicability of our model to those experiments rests basically on two assumptions. First, we assume that the strength of the short range attraction does not change with external parameters, and second, presently we have not gauged the interaction strength to that originating from multivalent counterion interactions. This important piece of work is left for future investigations. However, it appears plausible that at least for some parameter regions of biological charged polymers both assumptions are justified, so that our results can be used to provide a mechanism for finite bundle sizes.

Our results also demonstrate that the mean-field theory of Deserno [14 for a charged hydrophobic droplet looks qualitatively correct. Extending the Deserno analysis to charged sticky rods does not change the qualitative behavior [29].

\section{Acknowledgments}

We would like to thank M. Deserno, P. Pincus, H. Schiessel, and M. Tamashiro for stimulating discussions. This work was supported by the "Zentrum für Multifunktionelle Werkstoffe und Miniaturisierte Funktionseinheiten", grant BMBF 03N 6500, and the DFG within the SFB 625 and grant HO-1108/11-1.

\section{References}

[1] V. Bloomfield, Biopolymers 31, 1471 (1991).

[2] V. A. Bloomfield, Current Opin. Struct. Biol. 6, 334 (1996).

[3] C. Conwell, I. Vilfan, and N. Hud, Proc. Natl. Acad. Sci. (USA) 100, 9296 (2003).

[4] M. Bockstaller et al., Macromolecules 33, 3951 (2000).

[5] M. Bockstaller et al., Macromolecules 34, 6359 (2001).

[6] G. Wegner, Macromolecular chemistry and physics 204, 347 (2003).

[7] I. Rouzina and V. Bloomfield, J. Phys. Chem. 100, 9977 (1996).

[8] B.-Y. Ha and A. J. Liu, Phys. Rev. Lett. 81, 1011 (1998).

[9] Y. Levin, Rep. Prog. Phys. 65, 1577 (2002).

[10] B.-Y. Ha and A. Liu, Europhys. Lett. 46, 624 (1999).

[11] J. F. Stilck, Y. Levin, and J. J. Arenzon, J. Stat. Phys. 106, 287 (2002).

[12] O. Lambert, L. Letellier, W. Gelbart, and J. Rigaud, Proc. Natl. Acad. Sci. (USA) 97, 7248 (2000).

[13] M. Henle and P. Pincus, to be published .

[14] M. Deserno, Eur. Phys. J. E 6, 163 (2001).

[15] M. J. Stevens, Phys. Rev. Lett. 82, 101 (1999).

[16] M. J. Stevens, Biophys. J. 80, 130 (2001).

[17] I. Borukhov et al., J. Chem. Phys. 117, 462 (2002).

[18] G. S. Grest and K. Kremer, Phys. Rev. A 33, 3628 (1986).

[19] T. Soddemann, B. Dünweg, and K. Kremer, Eur. Phys. J. E 6, 409 (2001).

[20] M. J. Stevens and K. Kremer, J. Chem. Phys. 103, 1669 (1995).

[21] U. Micka and K. Kremer, Europhys. Lett. 49, 189 (2000).

[22] H. J. Limbach and C. Holm, Comp. Phys. Comp. 147, 321 (2002).

[23] H. J. Limbach, C. Holm, and K. Kremer, Europhys. Lett. 60, 566 (2002).

[24] H. J. Limbach and C. Holm, J. Phys. Chem. B 107, 8041 (2003).

[25] L. Rayleigh, Philos. Mag. 14, 182 (1882).

[26] Y. Kantor, M. Kardar, and H. Li, Phys. Rev. E 49, 1383 (1994).

[27] A. V. Dobrynin, M. Rubinstein, and S. P. Obukhov, Macromolecules 29, 2974 (1996).

[28] M. Deserno, C. Holm, and S. May, Macromolecules 33, 199 (2000).

[29] M. N. Tamashiro and H. Schiessel, to be published . 